\title{
Strategy and Structure: A Learning Perspective and
}

\section{Analysis}

\author{
Eric W. Ford, Ph.D., MPH (Corresponding Author) \\ Chief Science Officer \\ Complex Task Assessment Solutions and Information Technology, LLC \\ \& Pedagogue, University of North Carolina Greensboro \\ 12 Winterberry Ct. Greensboro, NC 27455; USA \\ Tel: 806-787-3267Ｅ-mail: ewford@gmail.com
}

Rebecca Wells, Ph.D.

Professor, Texas A\&M University, College Station, TX

Tel: 979-458-2246Ｅ-mail: wells@srph.tamhsc.edu

Terrie C. Reeves, MBA, Ph.D.

Research Advisor, Digital Frontiers Consulting, Columbus, $\mathrm{OH}$

E-mail: tcreeves@att.net

Received: November 23, 2013 Accepted: December 14, 2013

doi:10.5296/ber.v4i1.4602 URL: http://dx.doi.org/10.5296/ber.v4i1.4602

\begin{abstract}
This paper integrates the strategy and structure constructs using organizational learning theory. Learning theory is refined by arraying strategic and structure constructs along a path dependent, concatenated, continuum that is congruent with the original model of strategy and structure originally proposed by Chandler. The model also enhances methodological rigor by increasing congruence between theory and statistic application. Findings indicate that a single construct of strategy and structure can be employed effectively in management research.
\end{abstract}

\section{Introduction}

The management literature has not used great precision in defining many common terms-particularly with respect to 'strategic management' (Ford, Ginter, \& Duncan, 2000; Leontiades, 1982; Ronda-Pupo \& Guerras-Martin, 2012). Often, strategic management is considered to have two parts-strategy and structure. Strategy is the activities that surround the determination of "basic long-term goals... and the allocation of resources necessary for carrying out these goals" (Chandler, 1962, p. 13). Structure variables encompass organizational 
design characteristics that are critical to pursuing different strategies. Therefore, strategy and structure measures are both integral to a common latent construct. Further, there is empirical evidence they should be treated as a single metric because: 1) there is their relationship is reciprocal or interdependent in nature (Fredrickson, 1986) and 2) although the concepts are intellectually distinct, they behave as a single construct with variables describing each aspect intermingled.

The current study models learning in a path dependent continuum using Miller's typology of six learning modes and learning theory. Specific variables were drawn from Miller and Friesen's (1984) previous work on organizational configurations, later reflected in his(1996) learning typology. Item response theory (IRT) is used to analyze data from seventy-seven organizations' performance on the configuration variables. Both the organizations and the variables are arrayed along a common single dimension that reflects a learning continuum.

The study makes a unique contribution to strategy literature by putting both strategy and structure variables onto a continuous array that indicates a capability path. Capability development is inherently a learning exercise and is consistent with the organizational learning perspective (Schiller, 2014). Therefore, for strategy researchers, the ability to array strategy and structure measures along a single continuum is important as it simplifies many analyses. For practitioners, the results provide a defined path to assess and develop strategic capabilities in a learning fashion.

\section{Organizational Learning: Methodical and Emergent Constructs}

Organizational learning theory has been popular in management research because of the intuitive appeal of the concept that organizations, like people, can use information to change their behavior, and by so doing, may enjoy a longer, more productive existence. The analogies of organizational to individual learning may include the necessity of mastering one set of competencies before progressing to others that build on the foundation skills (Kusunoki, Nonaka, \& Nagata, 1998). Thus, learning is posited to be a combinative achievement (Kogut \& Zander, 1992), in which organizations master capabilities that are more fundamental before acquiring more complex strategic capacity.. Further, learning is the developmental process of modifying an organization's "cognitive map" (Friedlander, 1983: 194) using organizational capabilities, thereby expanding the range of potential strategic responses to an ever-changing environment (Fiol, 1996; Huber, 1991).

It is important to note that in order for learning to take place in organizations elements of both strategy and structure are required. First, organizations need structures and processes to consolidate knowledge that would otherwise remain inside the minds of individuals (Argote, 1999). Second, organizations rely upon shared norms, ideally a shared strategic vision, that facilitates the exchange of information and its incorporation into collective strategies. Popper and Lipshitz (2000: 183) describe both structural and cultural factors as organizational learning mechanisms, which they define as "institutionalized structural and procedural arrangements that allow organizations to systematically collect, analyze, store, disseminate, and use information relevant to the performance of the organization and its members." Sorensen (2002: 73) emphasizes the impact of learned organizational culture on organizational routines, but admits the possibility "that strong-culture firms may be better (or worse) at choosing appropriate strategies" (original parentheses). In contrast, this paper explores the organizational learning associated with successful strategizing. Although both culture and routines may be components of some strategic capabilities, the development of higher-level strategic capacity requires learning far beyond both. Understanding strategic capacity as a learning phenomenon allows us to probe its path dependent evolution based on previous work 
in both the organizational strategy and individual development fields.

Strategic capacity in term of organizational learning theory has been characterized as either "methodical" or "emergent" (Miller, 1996). Formalized strategy mechanisms and structures for focusing on specific goals and objectives within existing paradigms are methodical in nature (Steiner, 1997), while incremental approaches to strategy, like emergent learning, rely on more subtle normative considerations (Miller, 1996). Despite the proliferation of conceptual, theoretical, and empirical studies on strategy-environmental fit, debate continues to surround a key question: how do firms learn the strategic capabilities they need to succeed financially in their prevailing environments (Chan, Yung, \& Burns, 2000; Morgan \& Hunt, 2002)?

\subsection{Methodical Learning Modes}

Methodical learning employs rational analysis of data to make performance-optimizing decisions-an example being TQM. This is the model assumed by scientific management theory (Taylor, 1911) and is akin to "single loop" learning (Argyris \& Schon, 1996; Ashby, 1960).

\subsubsection{Structural Learning-Consciousness of Analysis and Adaptive Behaviour}

The first, most constrained mode of methodical learning Miller (1996: 494) identified was Structural, which he described as being "one of the most pervasive forms of methodical learning." Routines codify processes, enabling organizations to repeat sequences with a minimum of errors or effort. Such routines also encode values that guide how organizations learn. Thus, structural learning shapes both what organizations absorb and what is filtered out.

Two capabilities from Miller and Friesen's (1984) previous work best reflect structural learning-Consciousness of Analysis and Adaptation. Consciousness of Analysis is the beginning of all planning and entails reflection on both problems and arrays of potential solutions. In this sense, it is the precursor of all other strategic actions, even incremental adaptations. Adaptive behavior may then be build on analysis by responding appropriately to environmental conditions whose implications have been considered. In turn, appropriate patterns of adaptation may precede the ability to conduct and learn from experiments.

\subsubsection{Experimental Learning-Scanning and Control}

The second mode of methodical thought and action Miller (1996) described was Experimental, portrayed as learning through problem-driven searches for better solutions. Experimentation is central to Total Quality Improvement, which assumes that organizations increase their efficiencies through a continuous cycle of incremental, data-driven trials (Deming, 1982). Like Structural Learning, Experimental Learning builds on assumptions of rationality and planned change. However, in this mode there is more action as new ideas are provisionally implemented.

The two activities that best illustrate experimental learning are Scanning, or the exploration of the firm's environment for problems and opportunities, and Control, the mechanisms through which organizations learn about how well their existing routines are working (Miller \& Friesen, 1984). Methodical use of Scanning and Control reduces the "cognitive burdens of top managers" (Miller, 1996: 493) and does not require long-term planning in the way more complex forms of organizational learning do. In fact, learning how to experiment on a small scale may be a precondition for predicting future consequences of larger decisions.

\subsubsection{Analytic Learning-Futurity of Planning}

The third and least restrictive mode of methodical learning is Analytic, the deliberate, systematic assessment of current decisions on the future of the organization. Miller (1996) 
specifically pointed to the type of strategic, 'long-range,' planning promulgated by Allison, Ansoff, and Steiner $(1971 ; 1965 ; 1997$, respectively) as characterizing this mode. According to Steiner, (1997: 14), Futurity is "the essence of strategic planning." Miller (1996) portrays the Analytic mode as the highest in voluntarism. Another implication is that this mode is the most challenging among the methodical modes, and builds upon Structural and Experimental competencies. Moreover, all three methodical modes set the stage for the more spontaneous, creative emergent strategy development. Such a progression would be consistent with Brews and Hunt's (1999: 903) findings that "formal specific planning may be a necessary precursor to successful...emergent strategy (Kusunoki et al.)."

\subsection{Emergent Learning Modes}

Emergent or "double loop" learning, in contrast to methodical learning, is more intuitive, unstructured, and global in nature, and entails questioning underlying assumptions (Argyris \& Schon, 1996).

\subsubsection{Institutional Learning-Technocratization and Innovation}

The most constrained emergent mode, Institutional, is a form of organizational learning in which members respond to the normative and symbolic logics of their contexts (DiMaggio \& Powell, 1983). Such influences may become more powerful within organizations when higher proportions of members have professional identities and thus a common, profession-based ethos for action. As a critical mass of professional employees coalesces to shape strategic decisions, the process is described as Technocratization (Miller \& Friesen, 1984). Technocratization can facilitate Innovation as professionals look to their peers at other organizations for guidance about emerging strategic options.

\subsubsection{Interactive Learning-Integration and Risk taking}

The second emergent thinking mode, Interactive, occurs when organizations discover new opportunities from the conflicting objectives of their members (Miller, 1996). Two factors indicate Interactive Learning capabilities. The first is Integration of Decisions, which occurs when units across the organization complement and support other units. The second is the Risk Taking necessary to effect larger changes in organizational strategy implied by paradigm shifts. Together, these complementary factors make it possible for organizations to learn from new perspectives, even when they conflict with the existing patterns of voluntary behavior. Further, when Interactive Learning becomes the norm it may facilitate the mode posited to be the most challenging of all, Synthetic Learning.

2.2.3 Synthetic Learning-Communication, Strategic Reappraisal, and Decentralization of Strategy-Making Authority

The final, least restrictive mode of emergent thinking, Synthetic, is the intuitive, holistic incorporation of new elements into models for action (Nonaka, 1995). Synthetic Learning is the hardest learning mode because it requires the ability to discern patterns among seemingly unrelated phenomena. Although Miller (1996: 492) asserts that "synthesis is normally the product of a single creative mind," in fact, the complexity of organizations' internal and external contexts is arguably too great for even a small group to understand fully. The first element of Synthesis is the Communication flow throughout the organization necessary to transcend individual understanding and develop collective intelligence. At its highest level, this reaches the level of dialogue, a deeply attentive exchange "all but lost to the modern world" (Senge, 1990: 239). Dialogue may take the participants in completely unplanned directions as each voice builds on and responds to previous voices (Bakhtin, 1986). The purpose is to go beyond any one individual so that members can find new, shared meanings, 
rather than simply defend their initial positions. Senge (1990: 239) describes the benefit of such dialogue: "collectively, we can be more insightful, more intelligent than we can possibly be individually."

The second dimension of Synthesis is the Strategic Reappraisal necessary to engage in "double-loop" learning. Unless organizational members are willing to rethink both their strategies and the means of attaining them, they will be limited to improving performance within old paradigms like strong culture organizations who perform routines reliably, but cannot easily change routines (Sorensen, 2002). Synthesis-developing new meanings out of information-requires the ability to rethink old frameworks.

The final dimension posited to indicate Synthetic capacities is Decentralization of Strategy-Making Power. Once relevant information is disseminated throughout the organization and members become accustomed to questioning existing strategies, the highest level of learning can be attained. In decentralized organizations, strategic decision-making power is distributed throughout the organization rather than reserved for top management.

The developmental logic of methodical and emergent strategic capabilities in this framework suggests that a model of organizational learning should have path dependent characteristics. Therefore, the following hypothesis is made about the model's constructs:

Hypothesis 1: Methodical and emergent strategic capabilities fall along a single, unidimensional pathway.

\section{Research Design}

Concomitant with the development of a new strategy-environment fit model is the need for a method to empirically test its propositions while maintaining congruency between methods and vocabulary (Venkatraman, 1989). In trying to meet this need, this study employed two distinct steps and an appropriate empirical analysis was matched to each step. First was an examination of the developmental pathways of health service organizations' strategic capacities, using a set of latent trait analysis algorithms called Rasch analysis.

\subsection{Sample and Data}

Health care organizations were studied for three reasons. First, researchers studying the U.S. health system are beginning to call for and apply learning theory in studying both markets and management practices (Committee on Quality of Health Care in America, 2001; Kohn, Corrigan, \& Donaldson, 2000). This study answers those calls. Second, because the healthcare industry's environment is unstable due to rapid technological change, dramatic government interventions, and increasing competition (Gould, 1988), strategies and structures are under significant pressure to learn. Therefore, any learning pathway identified across organizations is indicative of the larger learning paradigm taking place. Finally, Ketchen et al., (1997) found that single industry studies produced greater effect sizes on variables related to strategy.

Study data were obtained from two types of sources: (a) Forms 10-K, prospectuses, and other forms filed with the Securities and Exchange Commission (SEC) and annual reports in the case of for-profit health services organizations, and (b) strategic management cases about health services organizations found in the following textbooks, Case Research Journal for the years 1990 through mid-1995; Harvard Business School 1994-1995 Catalog of Teaching Materials; Preferred Individualized Case (PIC) Catalog; the European Clearinghouse Catalog of cases; the Western Ontario Business School Teaching Materials Catalog; and finally the Darden Graduate School of Business Catalog of cases. All cases contained reference to most of the study's variables of interest. Organizations to be studied using SEC documents were chosen in 
the following manner. Based on the Standard Industrial Classification (SIC) codes for publicly traded health services organizations obtained from a national broker, a list of all other health services organizations with the same SIC codes was compiled. SEC documentation was gathered for those organizations about whom information was available in Standard and Poor's Corporate and Municipal Ratings, in Moody's Bond Record, or in Value Line, and whose major source of revenue appeared to be direct patient care. Raters were directed to pay special attention to descriptive sections about the external environment and strategies intended to deal with the environment. Raters looked for organizational responses to competitors, regulations, reimbursement mechanisms, or other external factors, and for internal mechanism that might facilitate responses. They were also instructed to read auditors notes to financial statements for clues about operating system such as new technological or computer systems, quality program, human resource practices. Fifty-seven usable sets of SEC documents were obtained. The others were unsuitable because the major source of revenue was not patient care related or data on all variables was unavailable. The final sample consisted of 20 cases and 57 sets of SEC documentation.

\subsection{Measures and Scoring}

Three sets of measures were developed to test the hypothesized relationship. First, a single measure of each organization's position on the strategic capacity continuum was calculated. Using variables operationalized in previous research (Miller \& Friesen, 1984), the strategic capacity measures used herein are based on the methodical and emergent organizational learning paradigms suggested by Miller (1996). Variables that failed to differentiate organizations in previous research or that did not have a direct counterpart in the learning paradigm descriptions were eliminated (See Appendix 1 for variable descriptions). In addition, the cues to scorers were modified for this study to reflect the health service context.

The variables were scored on a Likert scale with values ranging from one to seven. A value of one indicated that an organization lacked, or possessed very low amounts of a characteristic and a value of seven indicated that the organization had a significant amount of the capability. After extensive training sessions, multiple raters scored each organization's documents. All raters rated 43 percent of organizations studied. On 99.4 percent of those organizations' scores, evaluations varied by less than 2 between raters. Given this high level of agreement, scores were recorded as the average of the raters' scores in the few instances when the raters' scores differed.

Using a Rasch algorithm, a strategic capacity measure that Venkatraman (1989) would classify as using the 'fit as profile deviation' (p. 433) approach was developed. Here, differing levels of strategic capacity have been hypothesized as better suited for particular environmental conditions when better organizational performance is to be expected. Further, the concept of fit as profile deviation suggests that the degree of adherence to such a one-dimensional profile is related positively to performance. However, this study extended this analytical approach by using ipsative-scaling techniques to measure strategic capacity constructs. Ipsative scales' (similar to Guttman scaling) major characteristics are as follows: they are hierarchically cumulative (i.e., firms posses all of the strategic capabilities scoring at and below their score); they can detect small shifts in value; the scores are ordinal; they measure one dimension; and items are ordered in difficulty or complexity so that getting to one item implies success with the preceding item. In demanding an ordered array of items, ipsative scales are more rigorous than mere point estimates of associations because they define the entire range of ability not merely one level. As Brews and Hunt (1999, p. 890) describe, "These more sophisticated methodologies (see for example Fredrickson, 1984; Wood \& LaForge, 1979, 1981) have in 
general produced stronger planning/performance relationships than earlier work (Priem, Rasheed, \& Kotulic, 1995)." Further, by profiling all of the organizations together, rather than using a hold-out sample of successful organizations as Venktraman and Prescott (1990) did, the measure has more generalizability. The derived strategic capacity measure for each organization was then incorporated into interaction variables used in the second phase of the analyses related to hypotheses three through eight (a more detailed description of the algorithm is provided in the Analytic Approaches section).

\subsection{Analytic Approaches}

The analytic approaches used in this study contributed to theoretical and statistical correspondence in two ways. First, using expert raters to score variables on a Likert-like scale helps to overcome possible unequal scalar qualities among the concepts being rated. Second, Latent Trait Analysis (LTA) including Rasch models, is a family of procedures used to estimate a measure's dimensionality and the interval of ordinal-scaled items along a single dimension. First developed in the early 1960s for use in education and psychology, Rasch analysis models have been used to infer a person's position along a series of hierarchical items.

Rasch analysis can also identify items, such as strategy and structure measures, that are redundant and those that do not fit the model. In order to create an interval scale, Rasch analysis estimates both abilities (an organization's level of successful performance on a variable or capability) and item difficulty (level of resistance to successful performance) for a set of variables. The basic assumption is that the probability of an individual's or organization's success or failure on a particular item depends on both ability and the difficulty of the item.

The Rasch algorithm estimates item difficulty on a logistic scale in 'logits' (the log odds transformation of the probability of a correct response) and creates an interval scale. This technique can also identify characteristics or strategic capabilities that are redundant or that do not fit the presumed organizational learning pathway. The standardized infit (a weighted fit statistic) and outfit (an outlier sensitive fit statistic) statistics identify redundant items, noise, and outliers. Rasch users "routinely pay more attention to infit scores than outfit scores" (Bond $\&$ Fox, 2001, p. 43). Items with very low infit scores may be redundant. Items with unusually high infit scores indicate an unusual response pattern across items. For example, organizations with very high strategic capacity may lack an "easy," lower level capability. Such items can either be removed, if they are captured by another measure, or be retained if they are theoretically essential.

\section{Results}

Results of the Rasch procedure are presented in Table 1 . The twelve-item model had overall infit and outfit scores of $0.96(p<0.1)$ and $1.03(p<0.3)$ respectively with an item reliability of 0.71 (Cronbach's alpha). These measures indicate a good model fit. This finding supports hypothesis 1 that Miller's (1996) learning typology can be used to map strategic capacities along a single, unidemensional scale. Three of the items, Decentralization of Strategy-making, Innovation, and Adaptability / Proactivity were a significant distance from the central axis of the developmental pathway. Examining the points along the scale within each item, Decentralization of Strategy-making would have a better fit if it were reverse scored to measure centralization, thus putting it in the lower portion of the capacity scale. The other 11 strategic capacity measures were arrayed correctly, although two did not fit the model particularly well.

Table 1. Developmental Pathway Model of Strategic Management Dimensions and Diagnostics

\begin{tabular}{|l|l|l|l|}
\hline Item & Measure & Infit & Outfit \\
\hline
\end{tabular}




\begin{tabular}{|l|c|c|c|}
\hline De-centralization of Strategy- Making Power & 2.36 & $2.24^{*}$ & $2.98^{* *}$ \\
\hline Technocratization & 0.81 & 1.35 & 1.35 \\
\hline Strategic Reappraisal & 0.65 & 0.93 & 0.92 \\
\hline Communication & 0.04 & 0.75 & 0.78 \\
\hline Risk Taking & -0.04 & 1.16 & 1.14 \\
\hline Innovation & -0.17 & $0.63 \dagger$ & $0.62 \dagger$ \\
\hline Integration of Decision-making & -0.18 & 0.74 & 0.75 \\
\hline Control & -0.36 & 1.12 & 1.15 \\
\hline Scanning & -0.46 & 0.72 & 0.74 \\
\hline Futurity & -0.71 & 0.79 & 0.72 \\
\hline Adaptive Behavior & -0.83 & $0.50 \dagger$ & $0.49 \dagger$ \\
\hline Consciousness of Analysis & -1.11 & 0.67 & 0.68 \\
\hline
\end{tabular}

$\dagger \quad p<.1$

* $\quad p<.05$

** $\quad p<.01$

Within the portion of the scale addressing methodical capacity, only Futurity of Decision-making was not in the predicted order, falling lower in the scale than anticipated. Within the emergent learning portion of the scale, Technocratization appears to be a far more difficult capability to achieve than projected. Also, Integration of Decisions precedes Innovation, a reversal of the predicted order. Overall, the single continuum of methodical and emergent capabilities detected by Brews and Hunt (1999) is confirmed and further explicated with these results.

\section{Discussion}

In answering the first questions posed at the outset of this paper, "how do organizations develop their strategic capacities?" this study found that a learning pathway of strategic capacity does exist among the health care organizations studied. In keeping with Miller's (1996) learning capacity continuum, that pathway's course runs from methodical to emergent strategic capacity. In answering the second question, "Is a good 'fit' between an organization's strategic capacity and prevailing environmental conditions related to financial performance?" analyses indicated that interaction between strategic capacity and environmental conditions do influence financial performance in a variety of ways. In particular, mastering emergent strategic capabilities is correlated with better performance, compared to having mastered methodical strategic capabilities, in environments characterized as more hostile or more heterogeneous. When environmental dynamism is greater, relying on a methodical strategic capacity yields better financial performance.

\subsection{Strategic Capacity Pathways-Planning to Learn}

A strategic learning continuum exists among the health care organizations studied. Specifically, a developmental pathway of strategic capacity was found. Further, the study explicated the nature of the methodical-emergent capacity pathway finding synoptic planning to be a lower order strategic capability. Previous models, such as the Planning School, may provide sufficient explanations in some instances, but are not comprehensive.

Methodical strategic capacity is a necessary precondition to emergent capacity. This implies 
that firms must learn methodical capabilities before they can learn emergent capabilities, and that methodical capacity is the more easily acquired. A further implication is that organizations, having mastered all capabilities, may then be able to draw upon appropriate ones depending upon the situation. However, not every methodical or emergent capability fell as closely along the pathway as anticipated. Decentralization of Strategic Planning, Adaptive Behavior, and Innovation did not fit the model well. One possible explanation for this anomaly relates to a firm's place in the organizational life cycle. Constructs that are characteristic of entrepreneurial behaviors like these are found in young firms. Firms just starting out may not have had the time to develop methodical skills nor the history to engage in the sensemaking indicative of emergent strategies. Alternatively, it may be that entrepreneurial organizations must develop capacity all along the continuum quickly in order to survive; or perhaps entrepreneurial organizations must develop some strategic capabilities simultaneously with others. Another possible explanation is that these misfit capabilities are characteristics of prospector organizations. While Shortell and Zajac (1990) have shown that not all health services organizations are prospectors, due to rapid changes in the health services sector in recent years, more rapid acquisition of strategic capacities may be required for all health services organizations that survive. Forced into rapid strategic capacity acquisition, health services organizations have haphazardly acquired strategic capabilities, or for prospector firms, a separate pathway may exist that has innovation and adaptive behaviors as key stepping-stones. Nevertheless, even considering these deviations, the pathway described by the Rasch analysis closely matched the hypothesized ordering.

\subsection{Limitations}

Although the study strongly suggests both a path-dependent continuum of strategic capacity and significant implications of such capacity for financial performance in different environmental conditions, the cross-sectional nature of these data did not capture potential long-term financial performance benefits of adopting either more methodical or more emergent strategies under various environmental conditions. For instance, although greater emergent strategic capacity was negatively associated with financial performance, this begs the question of what the lagged effects might be. Does dynamism generally punish more innovative and organic strategic learning?-or are emergent learners rewarded later for investments that have negative affects now.? Similar issues arise relative to the other environmental dimensions examined. What are the long-term implications of investing in and learning more or fewer emergent capabilities in more and less heterogeneous and hostile environments over the long run? Longitudinal data will be necessary to determine how effects of different strategic learning levels relate to organizational performance over time. In addition, although health service providers constitute a diverse industry, the sample in this study included only direct service providers. There may be differences both in the progression of strategic capacity and its performance implications for firms in other service industries and for manufacturers.

\subsection{Areas of Future Research}

Based on this study, there appears to be a strong possibility that strategic capacity and environmental conditions interact in a variety of ways that affect financial performance. In 
particular, the relationship of environmental dynamism, strategic capacity level, and organizational financial performance. However, using longitudinal data, the dynamic developmental effects of increasing strategic capacity could be examined. This study sets the stage for such future work.

A second avenue for future research is an examination of the relative importance of each of the six modes of strategic capacity in organizational performance under varying environmental conditions. Based on this study, it appears that strategic capacities must be developed in a distinct order. For example, scanning the environment might be less valuable to organizations that have a marginally developed consciousness of analyses capability. It is analogous to being in a conversation where you can hear the other person, but do not speak the language. Equally interesting would be analysis of the importance of the strategic capacity level for different types of organizations: it may be that differences exist among organizations of different ages or of different sizes. Determining those differences would be of great benefit for practitioners.

Finally, this study looked at health care organizations whose major business is in the United States. Health services organizations doing business in other parts of the world may find that strategic capacity must be developed in a different order for better performance, or that a certain capacity interacts with environmental conditions differently. A study of differences between U.S. organizations and organizations from other countries would advance over-all understanding of strategic capacity's interaction with the environment around the world.

\section{References}

Allison, Graham T. (1971). Essence of Decision: Explaining the Cuban Missile Crisis. Boston: Brown Little.

Ansoff, H. Igor. (1965). Corporate Strategy: An Analytic Approach to Business Policy for Growth and Expansion. New York: McGraw-Hill.

Argote, Linda. (1999). Organizational learning: Creating, retaining and transferring knowledge. Boston: Kluwer Academic Publishers.

Argyris, Chris, \& Schon, Donald A. (1996). Organizational Learning II. New York: Addison-Wesley Publishing Company.

Arthur, Michael B. (1996). Careers in City Politics: The Case for Urban Democracy. Administrative Science Quarterly, 41(4), 726-728. http://dx.doi.org/10.2307/2393875

Ashby, William Ross. (1960). Design for a brain : The origin of adaptive behavior. New York: Wiley.

Bakhtin, M. M. (1986). Speech Genres and Other Late Essays (V. W. McGee, Trans.). Austin, TX: University of Texas Press.

Bond, Trevor G., \& Fox, Christine M. (2001). Applying the Rasch Model: Fundamental Measurement in the Human Sciences. Mahwah, N.J.: Lawrence Erlbaum Associates.

Chan, Joseph W.K., Yung, K.L., \& Burns, N.D. (2000). Environment-strategy fit: a study of 
Hong Kong manufacturing logistics. Logistics Information Management, 13(5), 286. http://dx.doi.org/10.1108/09576050010378513

Chandler, Alfred D., Jr. (1962). Strategy and structure: Chapters in the history of the American industrial enterprise. Cambridge, MA: MIT Press.

Committee on Quality of Health Care in America. (2001). Crossing the Quality Chasm: A New Health System for the 21 st Century. Washington, D.C.: National Academy Press.

Covin, J. G., \& Slevin, D. P. (1989). Strategic management of small firms in hostile and benign $\begin{array}{llll}\text { environments. Strategic } \quad \text { Management } \quad \text { Journal, } & \text { 10(1), }\end{array}$ http://dx.doi.org/10.1002/smj.4250100107

Deming, W. Edwards. (1982). Out of the crisis. Cambridge, MA: MIT Center for Advanced Educational Services.

Dess, Gregory G., \& Beard, Donald W. (1984). Dimensions of organizational task environments. Administrative Science Quarterly, 29, 52-73. http://dx.doi.org/10.2307/2393080

DiMaggio, Paul J., \& Powell, Walter W. (1983). The iron cage revisited: Institutional isomorphism and collective rationality in organizational fields. American Sociology Review, 48(2), 147-160. http://dx.doi.org/10.2307/2095101

Fiegenbaum, A., \& Thomas, H. (1990). Strategic groups and performance - the United States insurance industry, 1970-84. Strategic Management Journal, 11(3), 197-215. http://dx.doi.org/10.1002/smj.4250110303

Fiol, C. Marlene. (1996). Consensus, Diversity, and Learning in Organizations. In J. R. Meindl, C. Stubbart \& J. F. Porac (Eds.), Cognition within and Between Organizations (pp. 173-206). Thousand Oaks, CA: Sage.

Ford, Eric W., Ginter, Peter, M., \& Duncan, W. Jack. (2000). The confusing words of strategy: Improving teaching and research. Paper presented at the Southwest Academy of Management, San Antonio, TX.

Fredrickson, J. W. (1984). The comprehensiveness of strategic decision processes: Extensions, observations, future directions. Academy of Management Journal, 27(3), 445-466. http://dx.doi.org/10.2307/256039

Fredrickson, J. W. (1986). The strategic decision process and organizational structure. Academy of Management Review, 11(2), 280-298.

Friedlander, F. (1983). Patterns of Individual and Organizational Learning. In S. Srivastva (Ed.), The Executive Mind. San Francisco: Jossey-Bass.

Goll, I., \& Rasheed, A. M. A. (1997). Rational decision-making and firm performance: The moderating role of environment. Strategic Management Journal, 18(7), 583-591. http://dx.doi.org/10.1002/(SICI)1097-0266(199708)18:7<583::AID-SMJ907>3.0.CO;2-Z

Gould, Stephen J. (1988). Macrodynamic Trends in Health Care: A Distribution and Retailing 
Perspective. Health Care Management Review, 13(2), 15.

Huber, George P. (1991). Organizational learning: An examination of thecontributing processes and the literatures. Organizational Science, 2, 88-115. http://dx.doi.org/10.1287/orsc.2.1.88

Kogut, Bruce, \& Zander, Udo. (1992). Knowledge of the firm, combinative capabilities, and the replication of technology. Organization Science, 3(3), 383-397. http://dx.doi.org/10.1287/orsc.3.3.383

Kohn, Linda T., Corrigan, Janet M., \& Donaldson, Molla S (Eds.). (2000). To Err is Human: Building a Safer Health System. Washington, D.C.: National Academy Press.

Kusunoki, Ken, Nonaka, Ikujiro, \& Nagata, Akiya. (1998). Organizational capabilities in product development of Japanese firms: A conceptual framework and empirical findings. Organization Science, 9(6), 699-718. http://dx.doi.org/10.1287/orsc.9.6.699

Leontiades, Milton. (1982). The confusing words of business policy. Academy of Management Review, 7, 45-48.

Miller, Danny, \& Friesen, Peter H. (1984). Organizations: A quantum view. Englewood Cliffs, NJ: Prentice-Hall.

Miller, Danny. (1996). A preliminary typology of organizational learning: Synthesizing the literature. Journal of Management, 22(3), 485-505. http://dx.doi.org/10.1287/orsc.9.6.699

Morgan, Robert E, \& Hunt, Shelby D. (2002). Determining marketing strategy: A cybernetic systems approach to scenario planning. European Journal of Marketing, 36(4), 450. http://dx.doi.org/10.1108/03090560210417264

Nonaka, Ikujiro. (1995). The knowledge-creating company: How Japanese companies create dynamic of innovation. New York: Oxford University Press.

Popper, M., \& Lipshitz, R. (2000). Organizational learning: Mechanisms, culture, and feasibility. Management Learning, 31(2), 181-196. http://dx.doi.org/10.1177/1350507600312003

Priem, Richard L., Rasheed, Abdul M., \& Kotulic, A. G. (1995). Rationality in strategic decision processes, environmental dynamism and firm performance. Journal of Management, 21(5), 913-929. http://dx.doi.org/10.1177/014920639502100506

Ronda-Pupo, Guillermo Armando, \& Guerras-Martin, Luis Ángel. (2012). Dynamics of the evolution of the strategy concept 1962-2008: a co-word analysis. Strategic Management Journal, 33(2), 162-188. http://dx.doi.org/10.1002/smj.948

Schiller, Stefan. (2014). Financial Information Related to Dynamic Capabilities: The Corporate Innovation Platform. Research in Business and Management, 1(1), 2-27. http://dx.doi.org/10.5296/rbm.v1i1.4496

Senge, Peter M. (1990). The Fifth Discipline: The Art and Practice of the Learning 


\section{Macrothink}

Organization. New York: Doubleday Currency.

Sorensen, Jesper B. (2002). The strength if corporate culture and the reliability of firm performance. Administrative Science Quarterly, 47, 70-91. http://dx.doi.org/10.2307/3094891

Steiner, George A. (1997). Strategic Planning: What Every Manager Must Know. New York: Free Press.

Taylor, Frederick Winslow. (1911). The principles of scientific management (3rd ed.). New York: Harper and Brothers.

Venkatraman, N. (1989). The concept of fit in strategy research: Toward verbal and statistical correspondence. Academy of Management Review, 14(3), 423-444.

Wood, D. R. Jr., \& LaForge, R. L. (1979). The impact of comprehensive planning on financial performance. Academy of Management Journal, 22(3), 516-526. http://dx.doi.org/10.2307/255741

Wood, D. R. Jr., \& LaForge, R. L. (1981). Toward the development of a planning scale: An example from the banking industry. Strategic Management Journal, 2(2), 209-216. http://dx.doi.org/10.1002/smj.4250020209

\section{Copyright Disclaimer}

Copyright reserved by the author(s).

This article is an open-access article distributed under the terms and conditions of the Creative Commons Attribution license (http://creativecommons.org/licenses/by/3.0/). 